\title{
Assessment of diagnostic value of fluorescent lymphography-guided lymphadenectomy for gastric cancer
}

\author{
Minoa K. Jung ${ }^{1,2} \cdot$ Minah Cho ${ }^{1,3} \cdot$ Chul Kyu Roh ${ }^{1,3} \cdot$ Won Jun Seo ${ }^{1,3} \cdot$ Seohee Choi ${ }^{1,3} \cdot$ Taeil Son $^{1,3} \cdot$ Hyoung-II Kim ${ }^{1,3}$. \\ Woo Jin Hyung ${ }^{1,3}$
}

Received: 17 June 2020 / Accepted: 3 September 2020 / Published online: 18 September 2020

(c) The International Gastric Cancer Association and The Japanese Gastric Cancer Association 2020

\begin{abstract}
Background Indocyanine green fluorescent lymphography helps visualize the lymphatic drainage pattern in gastric cancer; however, it is unknown whether fluorescent lymphography visualizes all metastatic lymph nodes. This study aimed to evaluate the sensitivity of fluorescent lymphography to detect metastatic lymph node stations and lymph nodes and the risk of false-negative findings.

Methods Patients with clinical T1-4a gastric cancer were included. Indocyanine green was peritumorally injected the day prior to surgery by endoscopy. Gastrectomy with systematic D1+ or D2 lymphadenectomy was performed. Stations and lymph nodes were retrieved at the back-table using near-infrared imaging and classified as "fluorescent" or "non-fluorescent" and later matched with histopathological findings.

Results Among 592 patients who underwent minimally invasive gastrectomy from September 2013 until December 2016, lymph node metastases were present in 150 . The sensitivity of fluorescent lymphography in detecting all metastatic lymph node stations was $95.3 \%$ (143/150 patients), with a false-negative rate of $4.7 \%$ (7/150 patients) and the sensitivity in detecting all metastatic lymph nodes was $81.3 \%$ (122/150 patients). The negative predictive value was $99.3 \%$ for non-fluorescent stations and $99.2 \%$ for non-fluorescent LNs. For detecting all metastatic LN stations, subgroup analysis revealed $100 \%$ sensitivity for pT1a, $96.8 \%$ for pT1b, 100\% for pT2, 91.3\% for pT3, and $93.6 \%$ for pT4a tumors.

Conclusions Fluorescent lymphography-guided lymphadenectomy can be a useful method for radical lymphadenectomy by facilitating the complete dissection of all potentially positive LN stations. Fluorescent lymphography-guided lymphadenectomy appears to be a reasonable alternative to conventional systematic lymphadenectomy for gastric cancer.
\end{abstract}

Keywords Gastric cancer · Lymphadenectomy · Fluorescence · Indocyanine green

This work was presented in oral at the 13th International Gastric Cancer Congress IGCC 2019, 8-11 May 2019, Prague, Czech Republic.

Electronic supplementary material The online version of this article (https://doi.org/10.1007/s10120-020-01121-0) contains supplementary material, which is available to authorized users.

Woo Jin Hyung

wjhyung@yuhs.ac

1 Department of Surgery, Yonsei University College of Medicine, 50-1 Yonsei-ro, Seodaemun-gu, Seoul 120-752, Republic of Korea

2 Department of Surgery, Division of Visceral Surgery, University of Geneva, Geneva, Switzerland

3 Gastric Cancer Center, Yonsei Cancer Center, Yonsei University Health System, Seoul, Republic of Korea

\section{Introduction}

Complete metastatic LN resection through radical lymphadenectomy is fundamental for curative treatment of gastric cancer, because it frequently metastasizes to regional lymph nodes (LNs) [1-3]. Radical lymphadenectomy requires a thorough understanding of the perigastric lymphatic drainage network. The recommended extent of lymphadenectomy is based on the anatomy of the lymphatic system and depends on the incidence of metastatic LNs in different LN stations, based on the site of the primary tumor $[4,5]$. However, because LNs within the adipose tissue are difficult to identify through gross inspection, standard lymphadenectomy without visualizing the lymphatic system bears the risk of incomplete resection of potentially metastatic LNs [6]. These challenges in visualizing LNs in adipose tissue result in the resection of all LN-bearing soft 
tissues around vascular structures, nerves, and solid organs, potentially leading to complications including vascular injury or pancreatic fistulas $[7,8]$.

Various dyes and tracers have been assessed to visualize lymphatic drainage patterns from primary tumors. Recently, indocyanine green (ICG) has been used for near-infrared imaging to detect draining LNs during gastric cancer surgery [9-13]. ICG was initially intraoperatively injected peritumorally during sentinel node navigation surgery (SNNS) to identify the primary draining nodes in early-stage gastric cancer [14]. As opposed to SNNS, another method of fluorescent lymphography-guided lymphadenectomy involves peritumoral ICG injection one day before surgery, which has reportedly helped visualize the entire lymphatic pattern of all draining LNs of the primary lesion [15]. Consequently, fluorescent lymphography has facilitated complete lymphadenectomy, allowing for intraoperative assessment of the extent of lymphadenectomy and to retrieve LNs thoroughly from resected specimens [15]. However, it is unknown whether fluorescent lymphography detects all potentially metastatic LNs, mostly owing to a limited number of patients with metastatic cancers included in previous studies. The present study aimed to evaluate the diagnostic value of fluorescent lymphography with peritumoral ICG injection 1 day before surgery and to analyze its sensitivity in detecting metastatic LN stations and LNs during gastric cancer surgery.

\section{Methods}

\section{Patients}

A prospectively collected gastric cancer database to identify patients who underwent robotic or laparoscopic gastrectomy with fluorescent lymphography-guided lymphadenectomy between September 2013 and December 2016 was retrospectively reviewed. The inclusion criteria were as follows: (1) patients having histologically confirmed gastric adenocarcinoma, (2) patients with a single primary lesion, and (3) patients at a clinical stage of T1-4aN0-3M0. The exclusion criteria were as follows: (1) prior chemotherapy or radiation therapy for the current gastric cancer or (2) a previous gastric intervention, including endoscopic treatment. This study was approved by the institutional review board of Severance Hospital, Yonsei University College of Medicine (4-20180657), and informed consent was waived.

\section{Technique}

\section{Endoscopic ICG injection}

ICG was endoscopically injected peritumorally during routine endoscopic examination for tumor localization 1 day before surgery. ICG (Dongindang Pharmaceutical Co., Ltd., Siheung, Gyeonggi-Do, Korea) was used as the tracer, which was intraoperatively visualized using near-infrared optical systems for intraoperative navigation. Before 2015, $1.25 \mathrm{mg} / \mathrm{mL}$ ICG was prepared in sterile water and $0.6 \mathrm{~mL}$ of the solution was injected into the submucosal layer at four points around the primary lesion, amounting to $3 \mathrm{mg}$ of ICG. Because the intensity of near-infrared fluorescence signals in the da Vinci ${ }^{\circledR}$ Xi System (Intuitive Surgical, Sunnyvale, CA, USA) and Pinpoint ${ }^{\circledR}$ endoscopic fluorescent imaging system (Novadaq, Mississauga, ON, Canada) was very high at $1.25 \mathrm{mg} / \mathrm{mL}$, the concentration of ICG was subsequently reduced to $0.625 \mathrm{mg} / \mathrm{mL}$ in 2015 , with ICG amounting to $1.5 \mathrm{mg}$. The feasibility of this technique has been previously reported at our institution [15].

\section{Fluorescent lymphography-guided lymphadenectomy}

Near-infrared fluorescent imaging was obtained through either the Pinpoint ${ }^{\circledR}$ endoscopic fluorescence imaging system for laparoscopic surgery or the Firefly ${ }^{\mathrm{TM}}$ mode of the da Vinci Si or Xi Surgical System for robotic surgery. Intraoperatively, the visualization wavelengths were frequently switched between white light and near-infrared imaging for fluorescent lymphography-guided lymphadenectomy. After completion of the lymphadenectomy, near-infrared imaging was switched on for final observation of residual fluorescent lymph nodes, and any remaining stained nodes were removed (Online Resource Figure 1). D1+ lymphadenectomy was performed for patients with clinically early gastric cancer without suspicion of nodal metastases, and D2 lymphadenectomy was performed for patients with advanced gastric cancer or any suspicion of nodal metastases in accordance with the Korean Practice Guideline for Gastric Cancer [16]. The standard surgical procedures at our institution have been previously described $[17,18]$. During lymphadenectomy, all fluorescent and non-fluorescent LNs within D1+ or D2 lymphadenectomy dissection areas were resected. LNs beyond the D2 area were not resected, regardless of the presence of fluorescent LNs, except for the \#14v station.

\section{Postoperative LN harvest}

After fluorescent lymphography-guided lymphadenectomy, each LN station was first separated ex vivo from the resected specimen in accordance with the definitions of the Japanese classification of gastric carcinoma [19]. Thereafter, LN stations and LNs were examined for fluorescence directly through near-infrared imaging. LNs were classified as "fluorescent" if they were stained with ICG and "nonfluorescent" if they were not stained with ICG. Stations containing fluorescent LNs were classified as "fluorescent" 
stations, while those without fluorescent LNs were classified as "non-fluorescent" stations. The presence and absence of fluorescent staining were subsequently matched with the results of histopathological analysis. True-positive (TP) stations were defined as fluorescent stations observed through near-infrared imaging, which contained metastatic LNs on histopathological analysis. False-negative (FN) stations were defined as non-fluorescent stations observed through near-infrared imaging, which contained metastatic LNs on histopathological examination. True-positive LNs were defined as fluorescent LNs observed through near-infrared imaging, which were metastatic on histopathological assessment. False-negative LNs were defined as non-fluorescent LNs observed through near-infrared imaging, which were metastatic on histopathological examination. We finally re-confirmed the congruency between near-infrared imaging and histopathological examination of "fluorescent" and "non-fluorescent" LNs by examining paraffin blocks of the LNs through near-infrared imaging.

\section{Statistical analysis}

The sensitivity, specificity, false-negative rate, negative predictive value (NPV), and positive predictive value (PPV) of fluorescent lymphography for detecting LN metastases based on stations and LNs were evaluated. These proportions were determined by matching the presence or absence of fluorescence staining in stations or LNs with the results of histopathological analysis. First, the sensitivity of fluorescent lymphography was determined from the total number of patients with LN metastases. Therefore, the sensitivity of ICG fluorescent lymphography for detecting LN metastasis in stations or LNs was determined using the following formula: [TP patients with all metastases in fluorescent stations or LNs/(TP patients + FN patients with metastases outside fluorescent stations or LNs)]. Second, the diagnostic value of ICG fluorescent lymphography was determined on the basis of the total number of stations and LNs. Sensitivity was determined by dividing the number of TP stations or LNs by the number of TP plus FN stations or LNs $[\mathrm{TP} /(\mathrm{TP}+\mathrm{FN})]$. Specificity was determined by dividing the number of TN stations or LNs by the number of TN plus FP stations or LNs [TN/(TN + FP)]. PPV was determined by dividing the number of TP stations or LNs by the number of TP plus FP stations or LNs [TP/TP + FP]. NPV was determined by dividing the number of TN stations or LNs by the number of TN plus FN stations or LNs [TN/ $(\mathrm{TN}+\mathrm{FN})]$.

Continuous variables are reported as median with interquartile range, and the Mann-Whitney $U$ test was used to detect differences. Categorical variables are reported as number and proportion, and the Chi-square test or Fisher's exact test was used to compare the differences in proportions depending on the application criteria.

A logistic regression model was used to assess the independent predictors of false-negative results. Sex, age, and body mass index (BMI) were included in the model, and the model was adjusted for all other univariate variables significantly associated with a false-negative result. A backward stepwise method was used to retain all variables with a $p$ value $<0.05$ in the final multivariate model. Logistic regression analysis was performed to determine the maximum likelihood estimates of odds ratios (ORs) and their 95\% CIs. Goodness-of-fit was evaluated using the Hosmer-Lemeshow test. The discriminant capacity of the model was evaluated by determining the area under the curve (AUC) and the associated $95 \%$ CI. A $p$ value of $<0.05$ was considered statistically significant. The IBM SPSS Statistics (Version 23 for Windows; IBM Corp., NY, USA) software package was used for all statistical analyses.

\section{Results}

In total, 619 patients underwent fluorescent lymphography-guided lymphadenectomy, of which, 21 patients with multiple lesions, 5 patients with prior gastric surgery, and 1 patient with a pT4b lesion were excluded from the study. The remaining 592 patients were included in the analysis. No complications related to ICG injection occurred in any patient. Demographic factors did not differ between the metastatic and non-metastatic groups with regard to age, sex, and BMI. Early gastric cancer was diagnosed preoperatively in 404 of $592(68.2 \%)$ patients, and 505 of 592 $(85.3 \%)$ patients underwent robotic gastrectomy. LN metastases were detected in 150 (25.4\%) patients, while no LN metastases were detected in the remaining $442(74.6 \%)$ patients (Table 1).

\section{LN retrieval and detection of LN metastases}

A total of 34,440 LNs were retrieved from 5342 dissected stations in 592 patients. Of these, 22,916 (66.5\%) LNs were fluorescent LNs in 4035 (75.5\%) fluorescent stations.

The median number of retrieved LNs per patient was 55 (IQR 43-70) including 36 (IQR 24-50) fluorescent LNs per patient, corresponding to a median number of 9 (IQR 8-10) dissected stations including 7 (IQR 5-8) fluorescent stations (Fig. 1a). The median numbers of harvested LNs and dissected stations were significantly higher in the 150 patients with $\mathrm{LN}$ metastases than in the 442 patients without LN metastases [58 (IQR 47-75) vs. 54 (IQR 43-68), $P=0.021$; 
Table 1 Clinical, surgical, and pathological characteristics of patients

\begin{tabular}{|c|c|c|c|c|}
\hline Characteristics & All patients $(n=592)$ & $\begin{array}{l}\text { Patients without } \mathrm{LN} \text { metastases } \\
(n=442)\end{array}$ & $\begin{array}{l}\text { Patients with LN metastases } \\
(n=150)\end{array}$ & $p$ value \\
\hline Sex, $n(\%)$ & & & & 0.523 \\
\hline Male & $329(55.6)$ & $249(56.3)$ & $80(53.3)$ & \\
\hline Female & $263(44.4)$ & $193(43.7)$ & $70(46.7)$ & \\
\hline Age, years, median (IQR) & $55(46-65)$ & $55(46-64)$ & $55(46-69)$ & 0.356 \\
\hline BMI, kg/m², median (IQR) & $23.2(21.3-25.1)$ & $23.2(21.4-25.1)$ & $22.8(20.9-25.0)$ & $0.419^{*}$ \\
\hline Tumor size, mm, $n(\%)$ & & & & $<0.001$ \\
\hline$<40$ & $441(74.5)$ & $361(81.7)$ & $80(53.3)$ & \\
\hline$\geq 40$ & $151(25.5)$ & $81(18.3)$ & $70(46.7)$ & \\
\hline Location, $n(\%)$ & & & & 0.008 \\
\hline Upper body & $87(14.7)$ & $54(12.2)$ & $33(22.0)$ & \\
\hline Middle body & $226(38.2)$ & $173(39.1)$ & $53(35.3)$ & \\
\hline Lower body & $278(47)$ & 215 (48.6) & $63(42.0)$ & \\
\hline Esophagus & $1(0.2)$ & $0(0.0)$ & $1(0.7)$ & \\
\hline Lauren classification, $n(\%)$ & & & & 0.206 \\
\hline Intestinal & $192(32.4)$ & $144(32.6)$ & $48(32)$ & \\
\hline Diffuse & $369(62.3)$ & $279(63.1)$ & $90(60)$ & \\
\hline Mixed & $31(5.2)$ & $19(4.3)$ & $12(8.0)$ & \\
\hline Surgical approach, $n(\%)$ & & & & 0.602 \\
\hline Laparoscopic & $87(14.7)$ & $63(14.3)$ & $24(16.0)$ & \\
\hline Robotic & $505(85.3)$ & $379(85.7)$ & $126(84.0)$ & \\
\hline Resection, $n(\%)$ & & & & 0.016 \\
\hline DG & $469(79.2)$ & $356(80.5)$ & $113(75.3)$ & \\
\hline TG & $85(14.4)$ & $53(12.0)$ & $32(21.6)$ & \\
\hline PG & $36(6.1)$ & $31(7.0)$ & $5(3.3)$ & \\
\hline PPG & $2(0.3)$ & $2(0.5)$ & $0(0.0)$ & \\
\hline Extent of lymphadenectomy, $n(\%)$ & & & & $<0.001$ \\
\hline $\mathrm{D} 1+$ & $355(60.0)$ & $312(70.6)$ & $43(28.7)$ & \\
\hline D2 & $237(40.0)$ & $130(29.4)$ & $107(71.3)$ & \\
\hline cT classification, $n(\%)$ & & & & $<0.001$ \\
\hline cT1 & $377(63.7)$ & $328(74.2)$ & $49(32.7)$ & \\
\hline cT2-T3 & $184(31.1)$ & $105(23.8)$ & $79(52.7)$ & \\
\hline cT4 & $31(5.2)$ & $9(2.0)$ & $22(14.7)$ & \\
\hline $\mathrm{cN}$ classification, $n(\%)$ & & & & $<0.001$ \\
\hline Negative & $478(80.7)$ & $392(88.7)$ & $86(57.3)$ & \\
\hline Positive & $114(19.3)$ & $50(11.3)$ & $64(42.7)$ & \\
\hline pT classification, $n(\%)$ & & & & $<0.001$ \\
\hline T1a & $245(41.4)$ & $232(52.5)$ & $13(8.7)$ & \\
\hline T1b & $162(27.4)$ & $131(29.6)$ & $31(20.7)$ & \\
\hline $\mathrm{T} 2$ & $66(11.1)$ & $37(8.4)$ & $29(19.3)$ & \\
\hline $\mathrm{T} 3$ & $75(12.7)$ & $29(6.6)$ & $46(30.7)$ & \\
\hline $\mathrm{T} 4 \mathrm{a}$ & $44(7.4)$ & $13(2.9)$ & $31(20.7)$ & \\
\hline $\mathrm{pN}$ classification, $n(\%)$ & & & & $<0.001$ \\
\hline No & $442(74.7)$ & $442(100)$ & $0(0.0)$ & \\
\hline N1 & $65(11.0)$ & $0(0.0)$ & $65(43.4)$ & \\
\hline $\mathrm{N} 2$ & $47(7.9)$ & $0(0.0)$ & $47(31.3)$ & \\
\hline N3 & $38(6.4)$ & $0(0.0)$ & $38(25.3)$ & \\
\hline
\end{tabular}

Values are mean \pm standard deviation (interquartile range)

$L N$ lymph node, $S D$ standard deviation, $B M I$ body mass index, $D G$ distal gastrectomy, $T G$ total gastrectomy, $P G$ proximal gastrectomy, $P P G$ pylorus-preserving gastrectomy, $c T$ clinical depth of invasion, $c N$ clinical lymph node involvement, $p T$ pathological depth of invasion, $p N$ pathological lymph node involvement

*Mann-Whitney $U$ test, otherwise Chi-square or Fisher's exact test 
10 (IQR 9-10) vs. 9 (IQR 8-10), $P<0.001$, respectively]. Furthermore, the median numbers of fluorescent LNs and fluorescent stations were significantly higher in patients with than in those without LN metastases [39 (IQR 28-55) vs. 35 (IQR 24-50), $P=0.027$; 7.5 (IQR 6-9) vs. 7 (IQR $5-8), P<0.001$, respectively] (Fig. 1b). Among 150 patients with metastases, the median numbers of metastatic LNs and stations per patient were 3 (IQR 1-7) and 2 (IQR 1-2), respectively.

\section{Diagnostic value of fluorescent lymphography}

In 143 of 150 patients with LN metastases, all metastases were observed in fluorescent stations only. In 7 remaining patients with LN metastases, metastases were detected in both fluorescent and non-fluorescent stations. No patient displayed metastases exclusively in non-fluorescent stations. The sensitivity for the detection of metastatic stations through fluorescent lymphography was 95.3\% (143/150), and the FN rate was $4.7 \%$ (7/150) (Table 2). Therefore, the diagnostic accuracy of ICG fluorescent lymphography was $98.8 \%$ (585/592). Based on the pathological depth of invasion, the sensitivity was $100 \%$ (13/13) for pT1a, $96.8 \%$ (30/31) for pT1b, 100\% (29/29) for pT2, 91.3\% (42/46) for pT3, and 93.6\% (29/31) for pT4a tumors (Table 2). The sensitivity for detecting metastatic stations was $98.0 \%(48 / 49)$ for cT1, 93.7\% (74/79) for cT2-cT3, and 95.5\% (21/22) for cT4a tumors. The sensitivity for the detection of metastatic stations was $97.7 \%$ (42/43) for clinically early gastric cancers and $94.4 \%$ (101/107) for advanced gastric cancers.

In 122 of 150 patients with LN metastases, all LN metastases were detected exclusively in fluorescent LNs. In 27 of 150 patients, metastatic LNs were located in both fluorescent and non-fluorescent LNs. Only one patient presented a single LN metastasis in a non-fluorescent LN (Table 2). The sensitivity for the detection of metastatic LNs was $81.3 \%$ $(122 / 150)$, the false-negative rate was $18.7 \%$ (28/150), and the diagnostic accuracy was $95.3 \%$ (564/592) (Table 2). The sensitivity for the detection of metastatic LNs was 95.9\% (47/49) for cT1 tumors, 78.5\% (62/79) for cT2-cT3 tumors, and 59.1\% (13/22) for cT4a tumors. The sensitivity was $100 \%(13 / 13)$ for pT1a, 93.5\% (29/31) for pT1b, $100 \%(29 / 29)$ for pT2, $67.4 \%$ (31/46) for pT3, and $64.5 \%$ (20/31) for pT4 tumors (Table 2). The sensitivity for detecting metastatic LNs was $95.4 \%$ (41/43) for clinically early gastric cancers and 75.7\% (81/107) for clinically advanced gastric cancers.

The NPV of a non-fluorescent station was 99.3\% (1298 nonfluorescent and non-metastatic stations/1307 non-fluorescent stations) in 592 patients. The PPV of a fluorescent station was
7.3\% (296 fluorescent and metastatic stations/4 035 fluorescent stations) in 592 patients (Table 3). The NPV of a non-fluorescent $\mathrm{LN}$ was $99.2 \%$ (11 428 non-fluorescent and non-metastatic LNs/11 524 non-fluorescent LNs) in 592 patients. The PPV of a fluorescent LN was 3.1\% (703 metastatic and fluorescent LNs/22 916 fluorescent LNs) in 592 patients (Table 4).

\section{Predictors of false-negative results}

The 7 patients with false-negative, metastatic non-fluorescent stations included 1 patient with pT1b, 4 patients with pT3, and 2 patients with pT4a tumors. The 28 patients with false-negative, metastatic non-fluorescent LNs included 2 patients with pT1b, 15 patients with pT3, and 11 patients with pT4a tumors. Additional data on details of the 28 patients with false-negative metastatic non-fluorescent LNs are given in Online Resource Table 1. On univariable analysis, cT classification $(p=0.001), \mathrm{cN}$ classification $(p=0.013)$, pT classification $(p=0.003)$, and lymphovascular invasion $(p=0.005)$ were significantly associated with false-negative results when we considered the 28 false-negative patients with metastatic LNs in non-fluorescent LNs (Table 5). On multivariable analysis, cT4a (OR 9.83, 95\% CI 1.76-54.94, $p=0.009$ ) and lymphovascular invasion (OR $5.68,95 \%$ CI 1.21-26.82, $p=0.028$ ) were independent predictors of false-negative results (Table 5). Multivariate logistic regression model had good discriminant capacity, with an AUC of 0.78 (95\% CI 0.70-0.85, standard error 0.040) and was considered adequate using the Hosmer-Lemeshow test $(p=0.649)$.

\section{Discussion}

Herein, when the sensitivity of fluorescent lymphography (based on the total number patients) was evaluated for detecting metastatic lymph nodes, the sensitivity was acceptably high (above 93\%) for pT1 and pT2 tumors but decreased below $70 \%$ for pT3 and pT4 tumors. However, the sensitivity of fluorescent lymphography for detecting metastatic LN stations was 95.3\%. Moreover, the negative predictive value of non-fluorescent stations (or LNs) not to contain metastasis was over $99 \%$.

The high sensitivity of fluorescent lymphography for detecting metastatic stations implicates that the visualized ICG stained stations have a high probability of containing all potentially metastatic LNs. Furthermore, the high negative predictive value of non-stained stations implicates that the risk of residual metastatic LNs would remain very low even if non-fluorescent stations were not resected during 

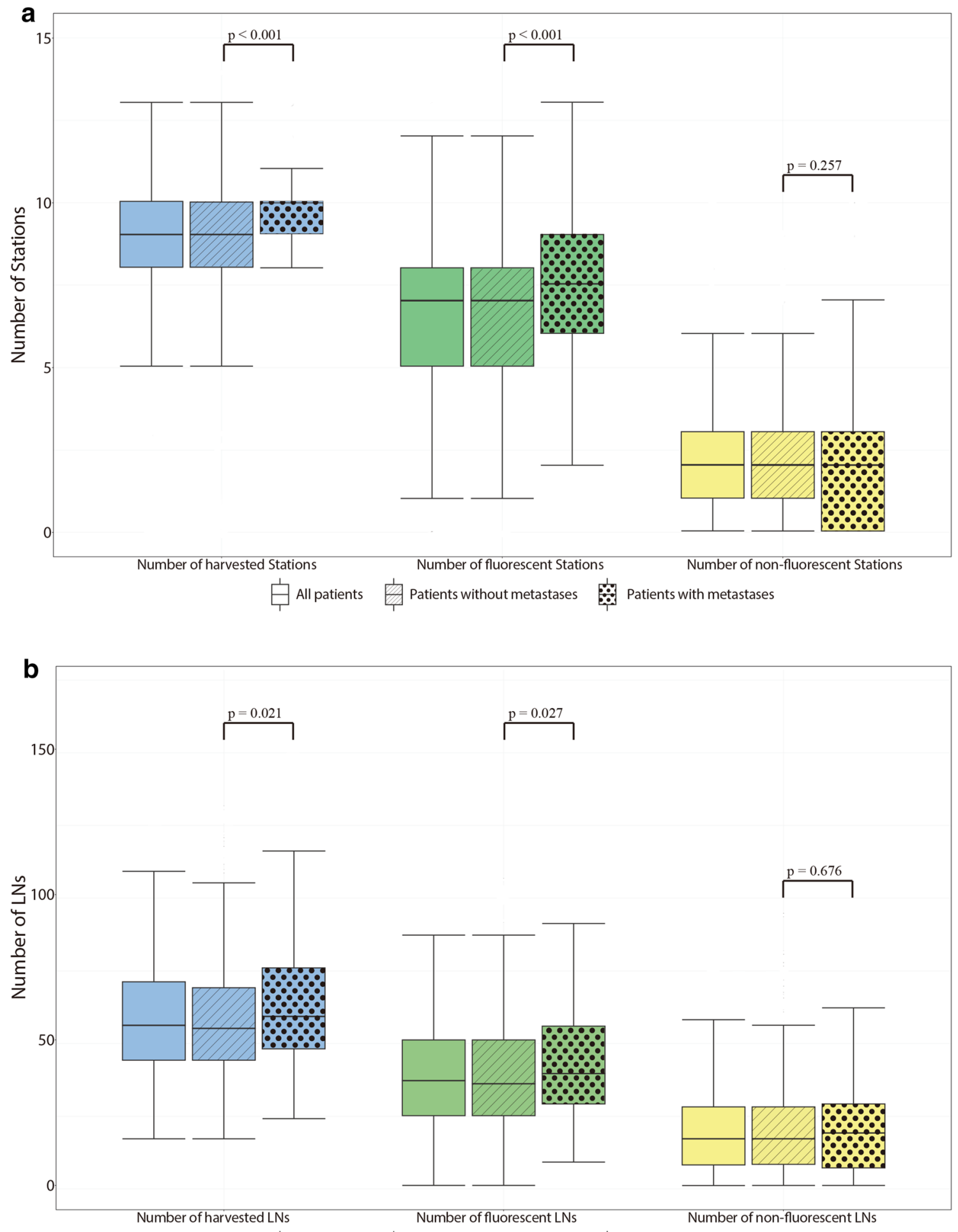

自All patients Patients without metastases Patients with metastases 
४Fig. 1 Fluorescent status of dissected lymph node stations and retrieved lymph nodes in patients with or without metastases. a Dissected lymph node (LN) stations per patient, with the fluorescent and metastatic station status in patients with or without metastases. The median number of dissected stations was significantly higher in the patients with LN metastases [10 (IQR 9-10)] than in the patients without LN metastases [9 (IQR 8-10); $P<0.001$ ]. The median number of fluorescent stations was also significantly higher in patients with LN metastases [7.5 (IQR 6-9)] than in patients without LN metastases [7 (IQR 5-8); $P<0.001$ ]. b Retrieved lymph nodes per patient, with fluorescent and metastatic lymph node status in patients with or without metastases. The median numbers of retrieved LNs was significantly higher in the patients with LN metastases [58 (IQR 47-75)] than in the patients without LN metastases [54 (IQR 43-68); $P=0.021]$. The median number of fluorescent LNs was also significantly higher in patients with LN metastases [39 (IQR 28-55)] than in patients without $\mathrm{LN}$ metastases [35 (IQR 24-50); $P=0.027$ ]

fluorescent lymphography-guided lymphadenectomy. Additionally, fluorescent lymphography helped guide surgeons for complete resection of the stained LN stations, thus decreasing the risk of damaging the contained LNs, thus preventing cancer cell spillage and bleeding. Thorough fluorescent lymphography-guided lymphadenectomy with high sensitivity and a low risk of residual metastatic stations reflected a high negative predictive value, also potentially resulting in an oncological benefit by increasing the curative effect of surgery. The present technique may also benefit the treatment of high-risk patients by reducing the surgery duration and decreasing morbidities associated with dissection around vessels and the pancreas. This technique may offer individualized lymphadenectomy, probably by waiving lymph node dissection of some stations which are not enhanced by this method. However, the false-negative rate should be as low as possible, and the false-negative rate of $4.7 \%$ in this ex vivo study could be acceptable, although it is not satisfactory. This technique might be particularly useful where the overall incidence of gastric cancer is low or when surgeons are confronted more frequently with advanced gastric cancer.

Studies on SNNS are aimed to treat node-negative earlystage gastric cancer of limited size by preserving organ function and limiting the extent of lymphadenectomy [10, 13, 20-23]. In contrast, our study aimed to perform complete lymphadenectomy through fluorescent lymphography. Thus, complete lymphadenectomy within the extent of D2 was performed regardless of fluorescent enhancement of the included LN stations herein. The present results indicate that fluorescent lymphography not only guides thorough lymphadenectomy but also has a high probability of detecting potentially metastatic LN stations. SNNS studies have reported high sensitivity when based on enhanced sentinel basins, although non-enhanced metastatic LNs were detected in up to $40 \%$ of patients [10]. Concurrent with SNNS studies, this study also revealed high sensitivity in detecting metastatic LNs in fluorescent stations. In contrast, the sensitivity for the detection of metastatic LNs among fluorescent LNs

Table 2 Sensitivity of fluorescent lymphography for the detection of metastatic stations and metastatic lymph nodes based on the total number of patients

\begin{tabular}{|c|c|c|c|c|c|c|c|c|}
\hline \multirow[t]{2}{*}{ pT stage } & \multicolumn{3}{|c|}{ Number of patients with metastases, $n(\%)$} & \multirow{2}{*}{$\begin{array}{l}\text { Sensitivity }{ }^{\mathrm{a}} \% \\
(\mathrm{CI})\end{array}$} & \multicolumn{3}{|c|}{ Number of patients with metastases, $n(\%)$} & \multirow{2}{*}{$\begin{array}{l}\text { Sensitivity }{ }^{\mathrm{b}} \% \\
\text { (CI) }\end{array}$} \\
\hline & $\begin{array}{l}\text { Fluorescent } \\
\text { stations only } \\
(\mathrm{TP})\end{array}$ & $\begin{array}{l}\text { Both fluores- } \\
\text { cent and non- } \\
\text { fluorescent } \\
\text { stations }\left(\mathrm{FN}^{1}\right)\end{array}$ & $\begin{array}{l}\text { Non-fluores- } \\
\text { cent stations } \\
\text { only }\left(\mathrm{FN}^{2}\right)\end{array}$ & & $\begin{array}{l}\text { Fluorescent } \\
\text { LNs only } \\
\text { (TP) }\end{array}$ & $\begin{array}{l}\text { Both fluores- } \\
\text { cent and non- } \\
\text { fluorescent } \\
\text { LNs }\left(\mathrm{FN}^{1}\right)\end{array}$ & $\begin{array}{l}\text { Non-fluores- } \\
\text { cent LNs only } \\
\left(\mathrm{FN}^{2}\right)\end{array}$ & \\
\hline All $(n=150)$ & $143(95.3)$ & $7(4.7)$ & $0(0)$ & $\begin{array}{l}95.3(90.6- \\
98.1)\end{array}$ & $122(81.3)$ & $27(18)$ & $1(0.7)$ & $\begin{array}{l}81.3(74.2- \\
87.2)\end{array}$ \\
\hline pT1a $(n=13)$ & $13(100)$ & $0(0)$ & $0(0)$ & $100(75.3-100)$ & $13(100)$ & $0(0)$ & $0(0)$ & $100(75.3-100)$ \\
\hline $\operatorname{pT} 1 \mathrm{~b}(n=31)$ & $30(96.8)$ & $1(3.2)$ & $0(0)$ & $\begin{array}{l}96.8(83.3- \\
99.9)\end{array}$ & $29(93.5)$ & $2(6.5)$ & $0(0)$ & $\begin{array}{l}93.5(78.6- \\
99.2)\end{array}$ \\
\hline pT2 $(n=29)$ & $29(100)$ & $0(0)$ & $0(0)$ & $100(88.1-100)$ & $29(100)$ & $0(0)$ & $0(0)$ & $100(88.1-100)$ \\
\hline pT3 $(n=46)$ & $42(91.3)$ & $4(8.7)$ & $0(0)$ & $\begin{array}{l}91.3(79.2- \\
97.6)\end{array}$ & $31(67.4)$ & $15(32.6)$ & $0(0)$ & $67.4(52-80.5)$ \\
\hline pT4 $(n=31)$ & $29(93.6)$ & $2(6.4)$ & $0(0)$ & $\begin{array}{l}93.6(78.6- \\
99.2)\end{array}$ & $20(64.5)$ & $10(32.3)$ & $1(3.2)$ & $\begin{array}{l}64.5(45.4- \\
80.8)\end{array}$ \\
\hline
\end{tabular}

$L N$ lymph node, pTla to pT4a pathological depth of invasion, $C I$ confidence interval $95 \%, T P$ true positive, $F N$ false negative

${ }^{\text {a }}$ Sensitivity for detection of metastatic stations was calculated by $\left[\mathrm{TP} / \mathrm{TP}+\left(\mathrm{FN}^{1}+\mathrm{FN}^{2}\right)\right]$

${ }^{\mathrm{b}}$ Sensitivity for detection of metastatic $\mathrm{LNs}$ was calculated by $\left[\mathrm{TP} / \mathrm{TP}+\left(\mathrm{FN}^{1}+\mathrm{FN}^{2}\right)\right]$ 
Table 3 Diagnostic value of fluorescent lymphography for the detection of metastatic stations based on the total number of stations

\begin{tabular}{|c|c|c|c|c|c|c|c|}
\hline pT stage & $\begin{array}{l}\text { Total } \\
\text { number of } \\
\text { stations }\end{array}$ & Metastatic stations & $\begin{array}{l}\text { Non- } \\
\text { metastatic } \\
\text { stations }\end{array}$ & Sensitivity $^{\mathrm{a}} \%(\mathrm{CI})$ & Specificity $^{\mathrm{b}} \%(\mathrm{CI})$ & $\mathrm{NPV}^{\mathrm{c}} \%(\mathrm{CI})$ & $\mathrm{PPV}^{\mathrm{d}} \%(\mathrm{CI})$ \\
\hline \multicolumn{8}{|l|}{ All } \\
\hline Fluorescent & 4035 & 296 (TP) & 3739 (FP) & $97.0(94.5-98.6)$ & $25.7(24.6-27.0)$ & 99.3 (98.7-99.7) & $7.3(6.6-8.2)$ \\
\hline Non-fluorescent & 1307 & $9(\mathrm{FN})$ & $1298(\mathrm{TN})$ & & & & \\
\hline \multicolumn{8}{|l|}{ pT1a } \\
\hline Fluorescent & 1586 & $16(\mathrm{TP})$ & 1570 (FP) & $100(79.4-100)$ & $24.5(22.7-26.4)$ & $100(99.3-100)$ & $1.0(0.6-1.6)$ \\
\hline Non-fluorescent & 510 & $0(\mathrm{FN})$ & $510(\mathrm{TN})$ & & & & \\
\hline \multicolumn{8}{|l|}{ pT1b } \\
\hline Fluorescent & 1041 & $50(\mathrm{TP})$ & $991(\mathrm{FP})$ & $98.0(89.6-100)$ & $27.0(24.7-29.5)$ & $99.7(98.5-100)$ & $4.8(3.6-6.3)$ \\
\hline Non-fluorescent & 368 & $1(\mathrm{FN})$ & $367(\mathrm{TN})$ & & & & \\
\hline \multicolumn{8}{|l|}{ pT2 } \\
\hline Fluorescent & 474 & 44 (TP) & $430(\mathrm{FP})$ & $100(92.0-100)$ & $26.9(23.3-30.6)$ & $100(97.7-100)$ & $9.3(6.8-12.3)$ \\
\hline Non-fluorescent & 158 & $0(\mathrm{FN})$ & $158(\mathrm{TN})$ & & & & \\
\hline \multicolumn{8}{|l|}{ pT3 } \\
\hline Fluorescent & 594 & $112(\mathrm{TP})$ & $482(\mathrm{FP})$ & $94.9(89.3-98.1)$ & $24.0(20.7-27.5)$ & 96.2 (91.9-98.6) & $18.9(15.8-22.2)$ \\
\hline Non-fluorescent & 158 & $6(\mathrm{FN})$ & $152(\mathrm{TN})$ & & & & \\
\hline \multicolumn{8}{|l|}{ pT4a } \\
\hline Fluorescent & 340 & 74 (TP) & $266(\mathrm{FP})$ & $97.4(90.8-99.7)$ & $29.4(24.9-34.3)$ & $98.2(93.8-99.8)$ & $21.8(17.5-26.5)$ \\
\hline Non-fluorescent & 113 & $2(\mathrm{FN})$ & $111(\mathrm{TN})$ & & & & \\
\hline
\end{tabular}

pTla to pT4a pathological depth of invasion, $C I$ confidence interval $95 \%, T P$ true positive, $F P$ false positive, $F N$ false negative, $T N$ true negative ${ }^{\mathrm{a}}$ Sensitivity was calculated by $[\mathrm{TP} / \mathrm{TP}+\mathrm{FN}]$

${ }^{\mathrm{b}}$ Specificity was calculated by $[\mathrm{TN} / \mathrm{TN}+\mathrm{FP}]$

${ }^{\mathrm{c}} \mathrm{NPV}$, negative predictive value was calculated by $[\mathrm{TN} / \mathrm{TN}+\mathrm{FN}]$

${ }^{\mathrm{d}} \mathrm{PPV}$, positive predictive value was calculated by $[\mathrm{TP} / \mathrm{TP}+\mathrm{FP}]$

was low in advanced tumors. Therefore, the present results suggest that station-based dissection is safer than the dissection of individual LNs. Hence, complete dissection of all fluorescent stations is recommended.

This technique may influence the extent of resection of the stomach because fluorescent lymphography helped visualize the specific lymphatic drainage pattern as a function of the individual tumor location. However, current recommendations regarding the extent of lymphadenectomy in gastric cancer do not consider the specific tumor location. For instance, a tumor located in the mid-body of the stomach can be treated with proximal, distal, or total gastrectomy, all of which involve different areas of lymphadenectomy. The possibility of leaving draining or connected LNs to the primary lesion undissected could increase if either distal or proximal gastrectomy is performed for a tumor located in the mid-body. Thus, fluorescent lymphography to visualize the distribution of all draining stations could provide useful information to determine the extent of gastric resection based on lymphatic anatomy. We investigated the high false negative rate in detection of metastatic LNs in pT3/pT4 tumors and found lymphovascular invasion and cT4 stage to be independent risk factors of false negative results in the 28 patients with LN metastasis in non-fluorescent LNs. It might be concluded that lymphovascular invasion obstructed the lymphatic ways and hindered the ICG from reaching each draining LN.

This study has some limitations. First, we could not determine the optimal timing and dose of ICG for ideal fluorescent lymphography, as different timings and doses were not evaluated. Our injection protocol for peritumoral submucosal injection of ICG 1 day before surgery helped visualize the entire lymphatic drainage pattern from the primary lesion. The interval between injection and operation should not be too short because ICG must be allowed to spread throughout the entire system of draining LNs. ICG staining was observed thereafter in lymphatic vessels [24, 25]. Future studies are required to establish the optimal injection protocol. Second, because oncological outcomes between conventional lymphadenectomy were not compared, we 
Table 4 Diagnostic value of fluorescent lymphography for the detection of metastatic lymph nodes based on the total number of lymph nodes

\begin{tabular}{|c|c|c|c|c|c|c|c|}
\hline pT stage & $\begin{array}{l}\text { Total } \\
\text { number } \\
\text { LNs, } n\end{array}$ & Metastatic LNs, $n$ & $\begin{array}{l}\text { Non-metastatic } \\
\text { LNs, } n\end{array}$ & $\begin{array}{l}\text { Sensitivity } \% \\
\text { (CI) }\end{array}$ & $\begin{array}{l}\text { Specificity }{ }^{\mathrm{b}} \% \\
\text { (CI) }\end{array}$ & $\mathrm{NPV}^{\mathrm{c}} \%(\mathrm{CI})$ & $\mathrm{PPV}^{\mathrm{d}} \%(\mathrm{CI})$ \\
\hline \multicolumn{8}{|l|}{ All } \\
\hline Fluorescent & 22,916 & $703(\mathrm{TP})$ & 22,213 (FP) & $88.0(85.5-90.2)$ & $34.0(33.5-34.5)$ & $99.2(99.0-99.3)$ & $3.1(2.8-3.3)$ \\
\hline Non-fluorescent & 11,524 & $96(\mathrm{FN})$ & $11,428(\mathrm{TN})$ & & & & \\
\hline \multicolumn{8}{|l|}{ pT1a } \\
\hline Fluorescent & 8842 & 21 (TP) & 8821 (FP) & $100(83.9-100)$ & $33.8(33.0-34.6)$ & $100(99.9-100)$ & $0.2(0.1-0.4)$ \\
\hline Non-fluorescent & 4507 & $0(\mathrm{FN})$ & $4507(\mathrm{TN})$ & & & & \\
\hline \multicolumn{8}{|l|}{ pT1b } \\
\hline Fluorescent & 6013 & $91(\mathrm{TP})$ & $5922(\mathrm{FP})$ & $95.8(89.6-98.8)$ & $34.3(33.3-35.3)$ & $99.9(99.7-100)$ & $1.5(1.2-1.9)$ \\
\hline Non-fluorescent & 3098 & $4(\mathrm{FN})$ & $3094(\mathrm{TN})$ & & & & \\
\hline \multicolumn{8}{|l|}{ pT2 } \\
\hline Fluorescent & 2697 & 79 (TP) & 2618 (FP) & $100(95.4-100)$ & $33.6(32.1-35.1)$ & $100(99.7-100)$ & $2.9(2.3-3.6)$ \\
\hline Non-fluorescent & 1325 & $0(\mathrm{FN})$ & $1325(\mathrm{TN})$ & & & & \\
\hline \multicolumn{8}{|l|}{ pT3 } \\
\hline Fluorescent & 3486 & 348 (ТP) & 3138 (FP) & $84.9(81.0-88.2)$ & $33.1(31.8-34.5)$ & $96.2(95.1-97.09$ & $10.0(9.0-11.0)$ \\
\hline Non-fluorescent & 1616 & $62(\mathrm{FN})$ & $1554(\mathrm{TN})$ & & & & \\
\hline \multicolumn{8}{|l|}{ pT4a } \\
\hline Fluorescent & 1878 & $164(\mathrm{TP})$ & $1714(\mathrm{FP})$ & $84.5(78.7-89.3)$ & $35.6(33.8-37.5)$ & $97.0(95.7-97.9)$ & $8.7(7.7-10.1)$ \\
\hline Non-fluorescent & 978 & $30(\mathrm{FN})$ & $949(\mathrm{TN})$ & & & & \\
\hline
\end{tabular}

$L N$ lymph node, pTla to $p T 4 a$ pathological tumor depth of invasion, $C I$ confidence interval $95 \%, T P$ true positive, $F P$ false positive, $F N$ false negative, $T N$ true negative

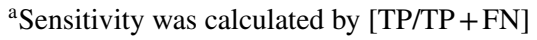

${ }^{\mathrm{b}}$ Specificity was calculated by $[\mathrm{TN} / \mathrm{TN}+\mathrm{FP}]$

${ }^{\mathrm{c}} \mathrm{NPV}$, negative predictive value was calculated by $[\mathrm{TN} / \mathrm{TN}+\mathrm{FN}]$

${ }^{\mathrm{d}} \mathrm{PPV}$, positive predictive value was calculated by $[\mathrm{TP} / \mathrm{TP}+\mathrm{FP}]$

could not evaluate the oncological efficacy of fluorescent lymphography. Third, it is not feasible to determine whether the high negative predictive value of non-fluorescent LNs stations indicates that the omission of non-fluorescent $\mathrm{LN}$ stations is safe, because we performed systematic lymphadenectomy regardless of fluorescent enhancement. Thus, future comparative studies between systematic lymphadenectomy and selective lymphadenectomy based on fluorescent lymphography are required. Moreover, our study revealed a low specificity and a very low positive predictive value in detecting metastatic LNs because ICG is not tumor specific. The development of tumor-specific tracers to investigate the feasibility of metastasis-specific and personalized lymphadenectomy for gastric cancer would be interesting. Fourth, we did not examine the dissected LNs through immunohistochemistry to evaluate the micrometastasis based on fluorescent enhancement. We intend to perform immunohistochemical assessment for micrometastasis based on fluorescent enhancement in future studies. Fifth, we did include lymphovascular invasion in our multivariate analysis of independent risk factors for false-negative results. However, this parameter cannot be obtained preoperatively, and its clinical usefulness is, therefore, limited. Lastly, we excluded patients who received neoadjuvant treatment; hence, it remains unclear whether our technique is applicable after neoadjuvant therapy.

In conclusion, fluorescent lymphography-guided lymphadenectomy with endoscopic ICG injection 1 day before surgery can be a potentially useful method for radical lymphadenectomy by facilitating the complete dissection of all potentially positive LN stations. Fluorescent lymphography-guided lymphadenectomy appears to be a reasonable alternative to conventional systematic lymphadenectomy for gastric cancer. Our results suggest the possibility of using this method for future clinical applications involving selective and individualized lymphadenectomy for gastric cancer patients. 
Table 5 Univariate and multivariate analyses of risk factors for false-negative results (non-fluorescent, metastatic lymph nodes) based on the total number of patients

\begin{tabular}{|c|c|c|c|c|c|c|c|}
\hline \multirow[t]{2}{*}{ Characteristics } & \multirow[t]{2}{*}{ Total number } & \multicolumn{3}{|c|}{ Univariable analysis } & \multicolumn{3}{|c|}{ Multivariable analysis } \\
\hline & & OR & $95 \% \mathrm{CI}$ & $p$ value & OR & $95 \% \mathrm{CI}$ & $p$ value \\
\hline \multicolumn{8}{|l|}{ Sex } \\
\hline Male & 329 & 1 & & & 1 & & \\
\hline Female & 263 & 1.406 & $0.617-3.204$ & 0.418 & 2.096 & $0.813-5.404$ & 0.126 \\
\hline \multicolumn{8}{|l|}{ Age, years } \\
\hline$<60$ & 392 & 1 & & & 1 & & \\
\hline$\geq 60$ & 200 & 2.120 & $0.924-4.863$ & 0.076 & 2.107 & $0.812-5.468$ & 0.126 \\
\hline \multicolumn{8}{|l|}{ BMI, $\mathrm{kg} / \mathrm{m}^{2}$} \\
\hline$<25$ & 435 & 1 & & & & & \\
\hline$\geq 25$ & 157 & 1.519 & $0.620-3.721$ & 0.360 & & & \\
\hline \multicolumn{8}{|l|}{ Tumor size, mm } \\
\hline$<40$ & 441 & 1 & & & & & \\
\hline$\geq 40$ & 151 & 1.179 & $0.518-2.681$ & 0.695 & & & \\
\hline \multicolumn{8}{|l|}{ cT classification } \\
\hline cT1 & 377 & 1 & & & 1 & & \\
\hline cT2-3 & 184 & 6.444 & $1.419-29.268$ & 0.016 & 3.757 & $0.773-18.260$ & 0.101 \\
\hline cT4a & 31 & 16.269 & $3.122-84.772$ & 0.001 & 9.832 & $1.760-54.938$ & 0.009 \\
\hline \multicolumn{8}{|l|}{$\mathrm{cN}$ classification } \\
\hline Negative & 478 & 1 & & & & & \\
\hline Positive & 114 & 2.974 & $1.264-6.995$ & 0.013 & & & \\
\hline \multicolumn{8}{|c|}{ Lauren classification } \\
\hline Intestinal & 192 & 1 & & & & & \\
\hline Diffuse & 369 & 1.250 & $0.499-3.131$ & 0.634 & & & \\
\hline Mixed & 31 & 1.000 & $0.183-5.460$ & 0.999 & & & \\
\hline \multicolumn{8}{|c|}{ Lymphovascular invasion } \\
\hline Absent & 451 & 1 & & & 1 & & \\
\hline Present & 141 & 8.432 & $1.913-37.169$ & 0.005 & 5.683 & $1.205-26.816$ & 0.028 \\
\hline
\end{tabular}

$O R$ odds ratio, $C I$ confidence interval, $B M I$ body mass index, $c T$ clinical tumor depth of invasion, $c N$ clinical lymph node involvement
Acknowledgements No funding was received for this study. We acknowledge the assistance of Wolters Kluwer, Editage (Trevose, PA, USA), with editing and correction of English language usage.

\section{Funding None.}

\section{Compliance with ethical standards}

Conflict of interest Dr. Hyung WJ received research grants from the Medtronic and GC Pharma and is the chief executive officer of Hutom and holding its stocks. He provided consultancy services to Ethicon and Verb Surgical outside the submitted work. Dr. Minoa K Jung received research grants from the Intuitive Surgical and received consulting fees from Johnson and Johnson, outside this project. No other disclosures were reported.

\section{References}

1. Ishikawa $\mathrm{S}$, Togashi $\mathrm{A}$, Inoue $\mathrm{M}$, Honda $\mathrm{S}$, Nozawa F, Toyama $\mathrm{E}$, et al. Indications for EMR/ESD in cases of early gastric cancer: relationship between histological type, depth of wall invasion, and lymph node metastasis. Gastric Cancer. 2007;10:35-8.

2. Mocellin S. The effect of lymph node dissection on the survival of patients with operable gastric carcinoma. JAMA Oncol. 2016;2:1363-4.

3. Songun I, Putter H, Kranenbarg EM, Sasako M, van de Velde CJ. Surgical treatment of gastric cancer: 15-year follow-up results of the randomised nationwide Dutch D1D2 trial. Lancet Oncol. 2010;11:439-49.

4. Maruyama K, Gunvén P, Okabayashi K, Sasako M, Kinoshita T. Lymph node metastases of gastric cancer. General pattern in 1931 patients. Ann Surg. 1989;210:596-602.

5. Tokunaga M, Ohyama S, Hiki N, Fukunaga T, Yamada K, Sano $\mathrm{T}$, et al. Investigation of the lymphatic stream of the stomach in gastric cancer with solitary lymph node metastasis. World $\mathrm{J}$ Surg. 2009;33:1235-9.

6. Roukos DH, Paraschou P, Lorenz M. Distal gastric cancer and extensive surgery: a new evaluation method based on the study of the status of residual lymph nodes after limited surgery. Ann Surg Oncol. 2000;7:719-26.

7. Yang SY, Roh KH, Kim YN, Cho M, Lim SH, Son T, et al. Surgical outcomes after open, laparoscopic, and robotic gastrectomy for gastric cancer. Ann Surg Oncol. 2017;24:1770-7. 
8. Yoshida K, Honda M, Kumamaru H, Kodera Y, Kakeji Y, Hiki N, et al. Surgical outcomes of laparoscopic distal gastrectomy compared to open distal gastrectomy: a retrospective cohort study based on a nationwide registry database in Japan. Ann Gastroenterol Surg. 2018;2:55-64.

9. Lee CM, Park S, Park SH, Jung SW, Choe JW, Sul JY, et al. Sentinel node mapping using a fluorescent dye and visible light during laparoscopic gastrectomy for early gastric cancer: result of a prospective study from a single institute. Ann Surg. 2017;265:766-73.

10. Kitagawa Y, Takeuchi H, Takagi Y, Natsugoe S, Terashima M, Murakami N, et al. Sentinel node mapping for gastric cancer: a prospective multicenter trial in Japan. J Clin Oncol. 2013;31:3704-10.

11. Kitagawa Y, Kubota T, Otani Y, Furukawa T, Yoshida M, Fujii H, et al. Clinical significance of sentinel node navigation surgery in the treatment of early gastric cancer. Nihon Geka Gakkai Zasshi. 2001;102:753-7.

12. Kitagawa Y, Ohgami M, Fujii H, Mukai M, Kubota T, Ando N, et al. Laparoscopic detection of sentinel lymph nodes in gastrointestinal cancer: a novel and minimally invasive approach. Ann Surg Oncol. 2001;8:86S-9S.

13. Miyashiro I, Hiratsuka M, Sasako M, Sano T, Mizusawa J, Nakamura K, et al. High false-negative proportion of intraoperative histological examination as a serious problem for clinical application of sentinel node biopsy for early gastric cancer: final results of the Japan Clinical Oncology Group multicenter trial JCOG0302. Gastric Cancer. 2014;17:316-23.

14. Mitsumori N, Nimura H, Takahashi N, Kawamura M, Aoki H, Shida A, et al. Sentinel lymph node navigation surgery for early stage gastric cancer. World J Gastroenterol. 2014;20:5685-93.

15. Kwon IG, Son T, Kim HI, Hyung WJ. Fluorescent lymphography-guided lymphadenectomy during robotic radical gastrectomy for gastric cancer. JAMA Surg. 2019;154(2):150-8.

16. Japanese Gastric Cancer A. Japanese gastric cancer treatment guidelines 2014 (ver. 4). Gastric Cancer. 2017;20:1-19.
17. Hyung WJ, Song C, Cheong JH, Choi SH, Noh SH. Factors influencing operation time of laparoscopy-assisted distal subtotal gastrectomy: analysis of consecutive 100 initial cases. Eur J Surg Oncol. 2007;33:314-9.

18. Song J, Kang WH, Oh SJ, Hyung WJ, Choi SH, Noh SH. Role of robotic gastrectomy using da Vinci system compared with laparoscopic gastrectomy: initial experience of 20 consecutive cases. Surg Endosc. 2009;23:1204-11.

19. Japanese Gastric Cancer A. Japanese classification of gastric carcinoma: 3rd English edition. Gastric Cancer. 2011;14:101-12.

20. Lee YJ, Jeong SH, Hur H, Han SU, Min JS, An JY, et al. Prospective multicenter feasibility study of laparoscopic sentinel basin dissection for organ preserving surgery in gastric cancer: quality control study for surgical standardization prior to phase III trial. Medicine (Baltimore). 2015;94(43):e1894.

21. Miyashiro I, Hiratsuka M, Kishi K, Takachi K, Yano M, Takenaka A, et al. Intraoperative diagnosis using sentinel node biopsy with indocyanine green dye in gastric cancer surgery: an institutional trial by experienced surgeons. Ann Surg Oncol. 2013;20:542-6.

22. Tajima Y, Yamazaki K, Masuda Y, Kato M, Yasuda D, Aoki T, et al. Sentinel node mapping guided by indocyanine green fluorescence imaging in gastric cancer. Ann Surg. 2009;249:58-62.

23. Becher RD, Shen P, Stewart JH, Geisinger KR, McCarthy LP, Levine EA. Sentinel lymph node mapping for gastric adenocarcinoma. Am Surg. 2009;75:710-4.

24. Landsman ML, Kwant G, Mook GA, Zijlstra WG. Light-absorbing properties, stability, and spectral stabilization of indocyanine green. J Appl Physiol. 1976;40:575-83.

25. Lim JS, Choi J, Song J, Chung YE, Lim SJ, Lee SK, et al. Nanoscale iodized oil emulsion: a useful tracer for pretreatment sentinel node detection using CT lymphography in a normal canine gastric model. Surg Endosc. 2012;26:2267-74.

Publisher's Note Springer Nature remains neutral with regard to jurisdictional claims in published maps and institutional affiliations. 